\title{
Video Article \\ Direct Pressure Monitoring Accurately Predicts Pulmonary Vein Occlusion During Cryoballoon Ablation
}

\author{
loanna Kosmidou ${ }^{1}$, Shannnon Wooden ${ }^{1}$, Brian Jones ${ }^{2}$, Thomas Deering ${ }^{1}$, Andrew Wickliffe ${ }^{1}$, Dan Dan ${ }^{1}$ \\ ${ }^{1}$ Department of Cardiac Electrophysiology, Piedmont Heart Institute \\ ${ }^{2}$ Cardiac Rhythm, Medtronic Inc.
}

Correspondence to: loanna Kosmidou at ioanna.kosmidou@piedmont.org

URL: https://www.jove.com/video/50247

DOI: doi: $10.3791 / 50247$

Keywords: Medicine, Issue 72, Anatomy, Physiology, Cardiology, Biomedical Engineering, Surgery, Cardiovascular System, Cardiovascular Diseases, Surgical Procedures, Operative, Investigative Techniques, Atrial fibrillation, Cryoballoon Ablation, Pulmonary Vein Occlusion, Pulmonary Vein Isolation, electrophysiology, catheterizatoin, heart, vein, clinical, surgical device, surgical techniques

Date Published: 2/26/2013

Citation: Kosmidou, I., Wooden, S., Jones, B., Deering, T., Wickliffe, A., Dan, D. Direct Pressure Monitoring Accurately Predicts Pulmonary Vein Occlusion During Cryoballoon Ablation. J. Vis. Exp. (72), e50247, doi:10.3791/50247 (2013).

\section{Abstract}

Cryoballoon ablation (CBA) is an established therapy for atrial fibrillation (AF). Pulmonary vein (PV) occlusion is essential for achieving antral contact and PV isolation and is typically assessed by contrast injection. We present a novel method of direct pressure monitoring for assessment of PV occlusion.

Transcatheter pressure is monitored during balloon advancement to the PV antrum. Pressure is recorded via a single pressure transducer connected to the inner lumen of the cryoballoon. Pressure curve characteristics are used to assess occlusion in conjunction with fluoroscopic or intracardiac echocardiography (ICE) guidance. PV occlusion is confirmed when loss of typical left atrial (LA) pressure waveform is observed with recordings of PA pressure characteristics (no $A$ wave and rapid $V$ wave upstroke). Complete pulmonary vein occlusion as assessed with this technique has been confirmed with concurrent contrast utilization during the initial testing of the technique and has been shown to be highly accurate and readily reproducible.

We evaluated the efficacy of this novel technique in 35 patients. A total of 128 veins were assessed for occlusion with the cryoballoon utilizing the pressure monitoring technique; occlusive pressure was demonstrated in 113 veins with resultant successful pulmonary vein isolation in 111 veins $(98.2 \%)$. Occlusion was confirmed with subsequent contrast injection during the initial ten procedures, after which contrast utilization was rapidly reduced or eliminated given the highly accurate identification of occlusive pressure waveform with limited initial training.

Verification of PV occlusive pressure during CBA is a novel approach to assessing effective PV occlusion and it accurately predicts electrical isolation. Utilization of this method results in significant decrease in fluoroscopy time and volume of contrast.

\section{Video Link}

The video component of this article can be found at https://www.jove.com/video/50247/

\section{Introduction}

Pulmonary vein isolation $(\mathrm{PVI})$ is a widely utilized invasive approach to treatment of atrial fibrillation. Standard PVI techniques include radiofrequency (RF) catheter ablation and, more recently, cryoablation utilizing a cryoballoon (Arctic Front, MDT Inc, MN, USA) ${ }^{1,2}$. Both techniques result in equal long-term efficacy in durable PVI and similar clinical outcomes ${ }^{3,4}$. Given the more rapid vein isolation achieved with the utilization of the cryoballoon, this latter approach has gained widespread acceptance by operators and is currently performed in most centers specializing in atrial fibrillation ablation.

Significant differences between the two techniques include, among others, the requirement of complete pulmonary vein (PV) occlusion with the cryoballoon in order to achieve effective electrical isolation. Apposition of the cryoballoon in the PV ostium is typically assessed fluoroscopically with the utilization of intravenous contrast. As such, the total amount of fluoroscopy utilized during cryoballoon ablation is higher compared to standard catheter ablation, thus increasing the risk of radiation injury. Further, risks associated with contrast infusion, such as renal injury and contrast allergy, are a significant limitation of this technique, given the frequent coexistence of chronic kidney disease in patients with atrial fibrillation ${ }^{5}$.

We present a novel method of direct PV pressure monitoring during cryoballoon inflation. This technique was validated in a total of 35 patients and reproducibility and accuracy was assessed. 
Protocol

\section{Methods/Procedure setup}

We included 32 patients with drug-refractory paroxysmal AF and three patients with persistent AF. Informed consent was obtained from all patients before the procedure.

\section{Ablation Procedure}

All patients are anticoagulated at least 1 month prior to the procedure. Warfarin is stopped the day prior to the procedure aiming for an international normalized ratio between 1.8 and 2.0 on the day of the procedure. Antiarrhythmic drugs are continued periprocedurally and for three months post procedure. All patients undergo cardiac MRI or cardiac CT imaging before the procedure to assess the left atrial and pulmonary vein anatomy and allow for measurement of PV diameter.

The ablation procedure is performed under conscious sedation. Electrograms are recorded in a specialized EP recording system (EP Med, St Jude Medical, MN, US).

\section{Procedure Setup}

1. The patient is draped in the standard way for ablation procedures. Vascular access is obtained in the right and left femoral veins. Systemic arterial pressure is continuously monitored via the left femoral artery through a pressure transducer. The intracardiac (ICE) ultrasound probe (Acuson, Siemens, GER) is advanced to the right atrium.

2. Using standard Fluoroscopic views and ICE guidance, trans-septal puncture is obtained using a Preface (Biosense Webster, CA) or SL1 sheath (St Jude Medical, MN). A J tipped 0.035" wire is placed in the left superior PV and the trans-septal sheath is exchanged with the Flexsheath. Before trans-septal access, heparin bolus is infused intravenously and then repeated as needed based on ACT checks performed every 20 min to maintain an activated clotting time over $350 \mathrm{sec}$ for the duration of the procedure.

3. Balloon size is selected in accordance with the diameter of the PVs based on cardiac MRI or cardiac CT measurements pre procedure. Typically, a $28 \mathrm{~mm}$ cryoballoon is utilized if any of the PV ostia has a diameter larger than $20 \mathrm{~mm}$.

4. After appropriate preparation, the cryoballoon is advanced over a wire or the Achieve catheter (Biosense Webster, CA) in the left atrium and then, using ICE and fluoroscopic guidance, into the antrum of each PV.

5. The balloon has an inner lumen that is typically used for radioiodine contrast injection through the distal tip of the balloon. A continuous pressure monitoring system is connected to the lumen to allow for pressure waveform analysis. Specifically, a 3-way Manifold (Navylist Medical, CA) (Figure 1) is attached via a Tuohy (Merit Medical, IRE) to the cryoballoon. Removal of air bubbles from the system is critical and care is taken to thoroughly flush the system prior to utilization. The three ports of the manifold are 1. saline flush (under pressure), 2. contrast and 3. pressure monitoring. The pressure monitoring port is then connected to a standard cardiac catheterization laboratory pressure transducer. The pressure waveforms are displayed on the EP recording system live monitor (Figure 2).

6. The LA pressure recordings are set at a scale of $25 \mathrm{mmHg}$ and sweep speed at $50 \mathrm{~mm} / \mathrm{sec}$. The page has minimal number of channels displayed (one or two surface ECG derivation, CS electrograms and arterial and LA pressure). Electrograms of the PV-LA junctions are recorded prior to ablation (either using the Achieve catheter or a separate multipolar catheter). Continuous recording is displayed on a separate page on the EP system when occlusion is assessed.

\section{PV Occlusion}

1. The deflated balloon is advanced over the guide-wire or Achieve catheter using standard fluoroscopic views and intracardiac ultrasound and is inflated on the LA side of the PV antrum.

2. The pressure page is displayed and the pressure line is opened to the transducer such that pressure is recorded from the tip of the balloon catheter (which dwells in the vein at this stage).

3. When the balloon does not occlude the ostium of the PV, a characteristic LA pressure is recorded. During sinus rhythm, $A$ (atrial) and $V$ (ventricular) waves are recorded with the $\mathrm{V}$ wave having a typical "isosceles triangle" morphology. During atrial fibrillation however, there is no consistent $A$ wave generated (since there is no atrial contraction) and only the $V$ wave morphology is seen. Using fluoroscopy or ultrasound guidance the balloon is advanced and placed in apposition with the PV ostium.

4. When occlusion is achieved, there is an abrupt change in the pressure waveform. During sinus rhythm there is a loss of the A wave and a change of amplitude (increase) and morphology of the $V$ wave. Since now the recording is that of trans-capillary pulmonary arterial pressure, the $\mathrm{V}$ wave has the typical characteristic of a more rapid rate of rise and a delayed downstroke. In fact the apex of the $\mathrm{V}$ wave triangle moves to the right when compared to the LA pressure $V$ wave recording. This is evident in sinus rhythm (Figure 3) and atrial fibrillation (Figure 4). This characteristic pressure waveform confirms complete occlusion and no further forward pressure or catheter manipulation needs to be used. Contrast use at this time may be utilized for further confirmation of PV occlusion during initial use of this methodology.

5. Once occlusion has been achieved, the freezing process can be started. During ablation of right-sided PVs, phrenic nerve pacing is performed from a catheter located in the superior vena cava in order to promptly detect phrenic nerve injury. For the first approximately $15 \mathrm{sec}$ (depending on local blood flow), pressure can still be recorded. Once temperature monitor reaches - 10 degrees Celsius, the inner lumen of the balloon freezes and pressure can no longer be recorded. At this stage, we toggle the recording system page to the intracardiac electrogram page to demonstrate "time to effect" of PV isolation. Cryoapplication is continued for $240 \mathrm{sec}$.

6. After the freezing process is complete and thawing has occurred, pressure can again be recorded and the maneuver can be repeated the number of times deemed necessary for permanent $\mathrm{PV}$ isolation.

7. After cryoablation, every PV is mapped using a 20-pole circular mapping catheter with variable diameter (Biosense Webster, CA). If remnant ostial potentials are still recorded, differential pacing of the atrium near the vein is performed to prove entrance block in the PV. In case of persistent conduction, electrical isolation is completed segmentally using an 8-mm cryoablation catheter (Freezor MaxR, Medtronic, MN) or 
an irrigated radiofrequency catheter (ThermoCool, Biosense Webster, CA). The endpoint of the procedure is complete electrical PV isolation, documented at least $30 \mathrm{~min}$ after the last application.

\section{Postablation Care}

After the procedure, warfarin is resumed and subcutaneous low molecular weight heparin is administered if necessary until the international normalized ratio is $\geq 2.0$. Warfarin is continued for at least 3 months. Clinical follow up was performed at six weeks, three months, six months and twelve months and included ECG at each visit and event monitoring at three months. Long term antiarrhythmic and anticoagulation therapy was determined by clinical outcomes.

\section{Representative Results}

We assessed the accuracy of pressure monitoring in predicting complete PV occlusion in 35 patients (28 males) with paroxysmal $(n=32)$ or persistent $(n=3)$ AF. Six patients had a prior AF ablation (17\%). A total of $128 \mathrm{PVs}$ were assessed with pressure monitoring during balloon inflation. Occlusive pressure was demonstrated with balloon inflation in $113 \mathrm{PVs}$, of which 111 were electrically isolated with CBA (98.2\%). Representative results are shown in Figure 5.

Lack of occlusive pressure waveform was more frequently noted in the RIPV (11/15 PVs). Two veins with demonstrated occlusive pressure waveform were still electrically connected despite concurrent demonstration of PV occlusion by contrast venography. The positive predictive value was $99 \%$. In the RIPV, we observed a requirement for up to three cryoapplications per vein when occlusive pressure was observed in $90 \%$ of RIPVs, whereas $10 \%$ of veins with occlusive pressure required 4 cryoapplications. In contrast, when occlusive pressure was not observed in the RIPV, 4 or 5 cryoapplications were performed in $70 \%$ of RIPVs.

A common PV ostium was observed in nine patients (27.1\%). Left common PV ostium was noted in eight patients and a right common PV in one patient. Occlusive pressure was achieved in 16/18 pulmonary veins with common ostium.

We observed a significant effect of the pressure monitoring technique in reducing total fluoroscopy time and radioiodine contrast utilization. Mean fluoroscopy time was $25.2 \pm 11.2 \mathrm{~min}$, mean contrast utilization $38 \pm 33 \mathrm{cc}$ and mean LA time $109 \pm 30.8$ min during cryoballoon ablation. Contrast and fluoroscopy time decreased significantly after the initial ten procedures utilizing the technique $(34.4 \pm 10.1 \mathrm{vs}$. $21.6 \pm 9.4 \mathrm{~min}$ of fluoroscopy, $p=0.001$ and $74 \pm 28$ vs $23.6 \pm 23 c c$ of contrast, $p=0.00$ ), indicating a very steep learning curve. No complications related to the utilization of the pressure monitoring technique were observed in this series of patients.

Post procedure arrhythmia free survival in one year was $60 \%$. Recurrence of AF or atrial flutter was noted in $40 \%$ of patients leading to repeat $\mathrm{PVI}$ in $35 \%$ of patients. $3 / 12$ patients with recurrent AF had previously documented persistent $\mathrm{AF}$ and $6 / 12$ patients had undergone $\mathrm{PVI}$ prior to the index procedure.

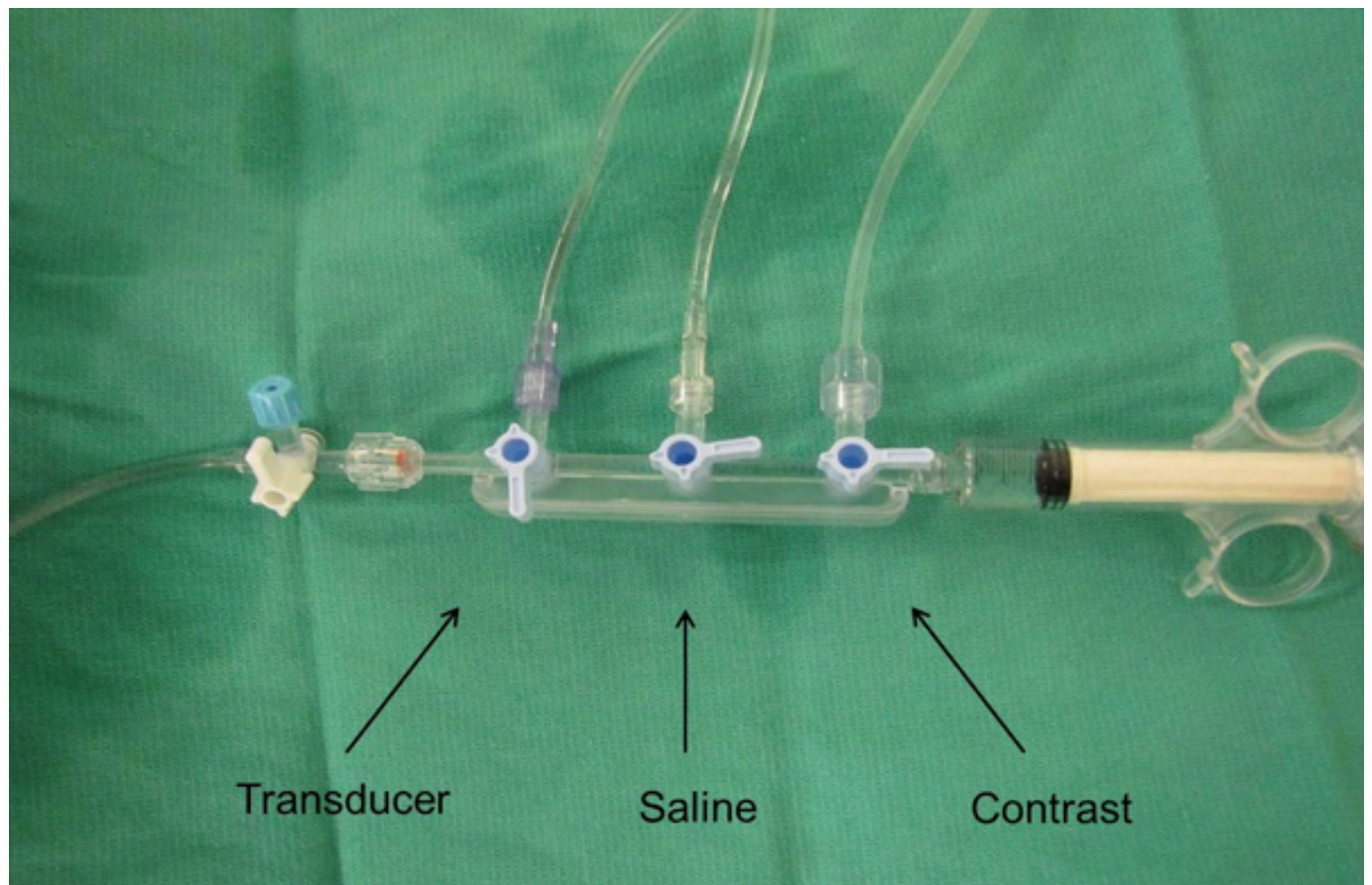

Figure 1. A 3-way manifold is connected via tubing to the inner lumen of the cryoballoon and a single pressure transducer for continuous pressure recording. 


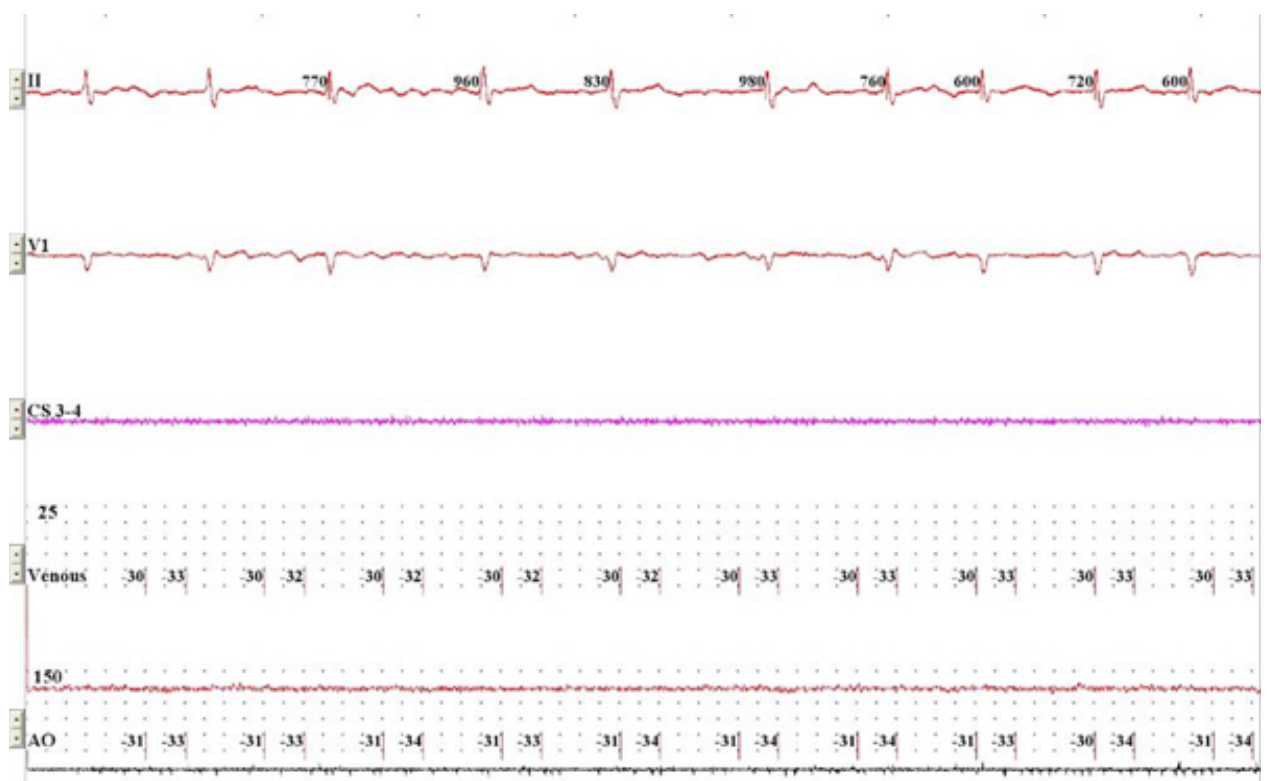

Figure 2. Standard monitoring screen during live recording. Surface ECG, intracardiac CS recording and venous and arterial pressure recording are displayed. Click here to view larger figure.

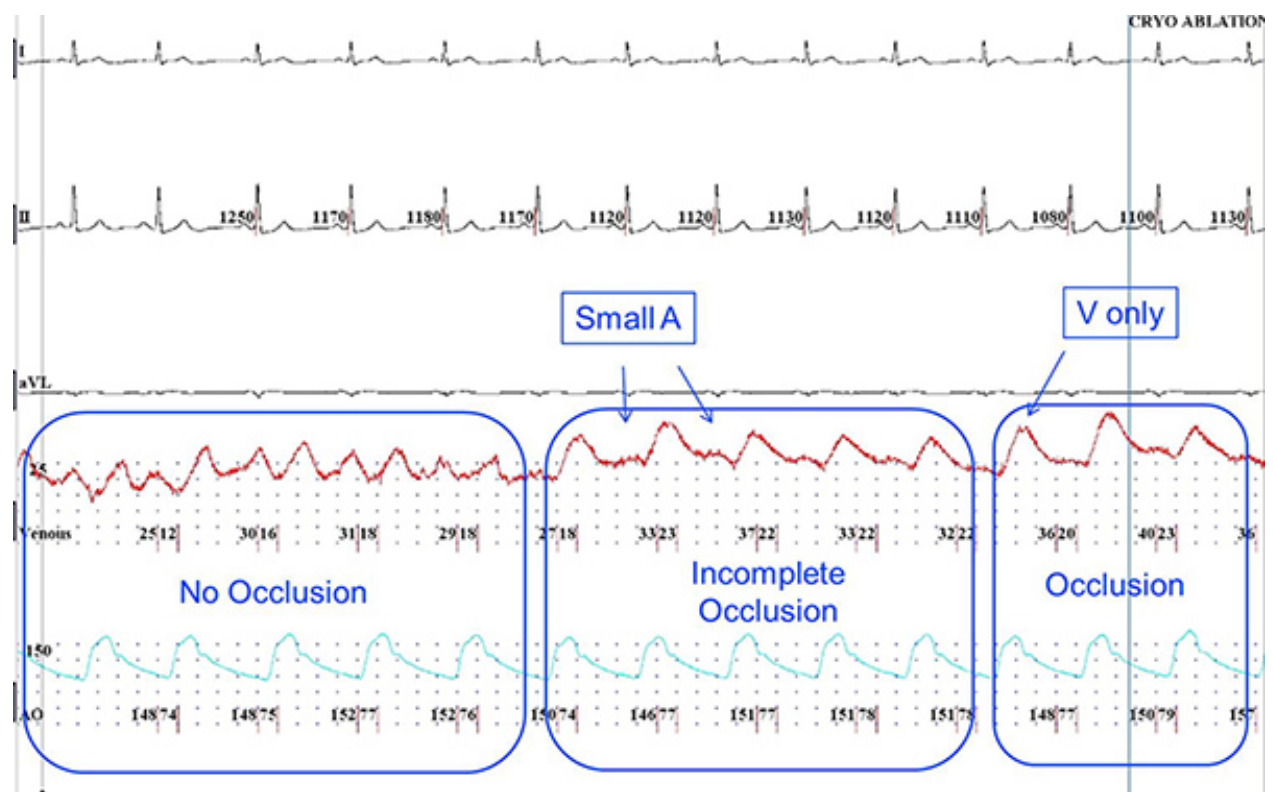

Figure 3. PV pressure waveform recording during sinus rhythm. Upon initial apposition of the deflated balloon (left panel), characteristic LA waveform is recorded with $A$ and $V$ waves noted. With initial inflation (middle panel), a change in the waveform is noted with more prominent $V$ waves and persistent lower amplitude A waves, suggesting incomplete venous occlusion. Further inflation and with successful circumferential apposition results in disappearance of the $\mathrm{A}$ wave and a larger $\mathrm{V}$ wave displaced towards mid diastole, confirming complete $\mathrm{PV}$ occlusion (right panel). Click here to view larger figure. 


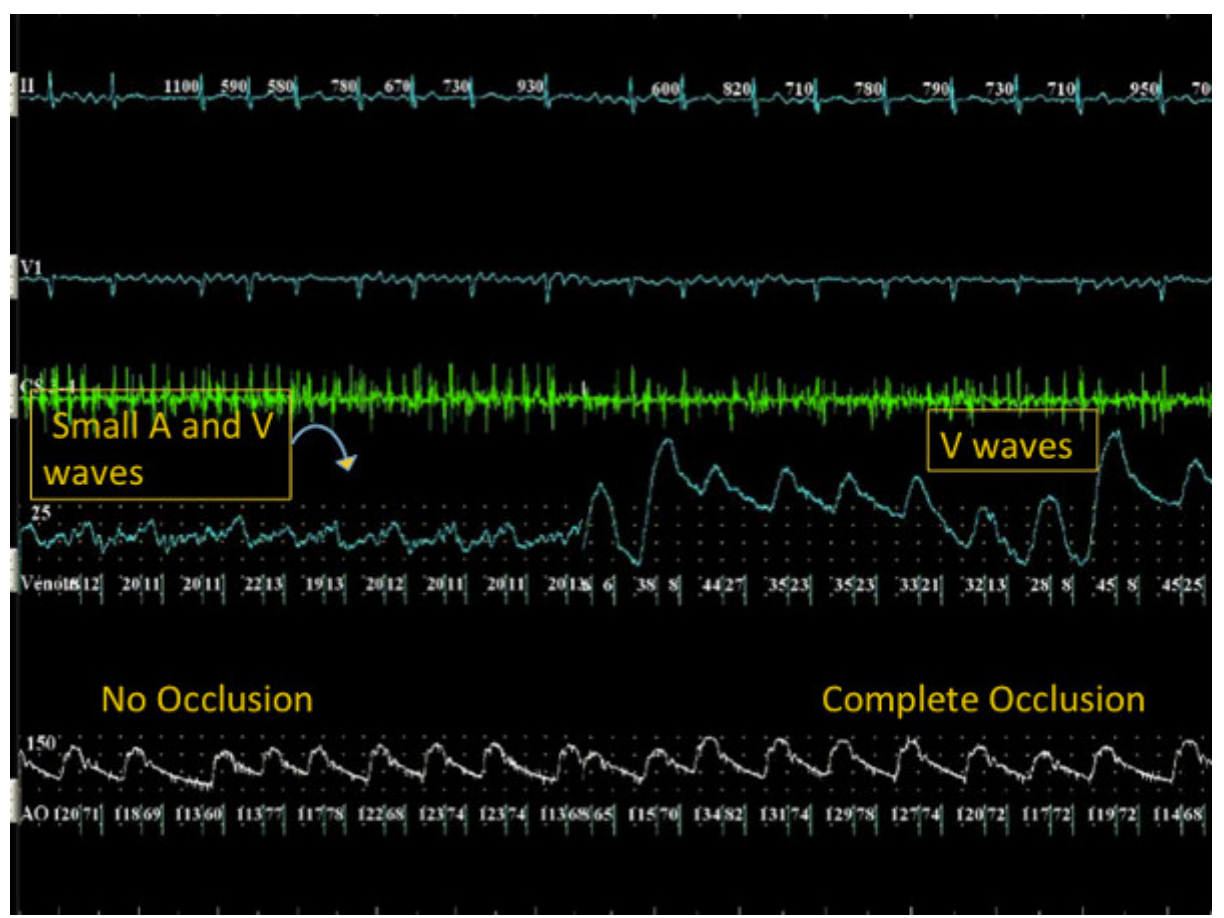

Figure 4. During atrial fibrillation, continuous atrial activity (A waves) with small amplitude $V$ waves is observed when the $P V$ is not occluded. With further manipulation of the cryoballoon in the PV ostium, abrupt increase in the $\mathrm{V}$ wave amplitude with loss of the small continuous atrial $\mathrm{A}$ waves is noted in the pressure waveform, confirming PV occlusion during atrial fibrillation. Click here to view larger figure.

\begin{tabular}{|l|l|}
\hline Total PVs, $n$ & 128 \\
\hline PVs with occlusive pressure, $n$ & $113 / 128$ \\
\hline $\begin{array}{l}\text { PVs isolated with CB when occlusive pressure is demonstrated }(\mathrm{n}) \text { and } \\
\text { PPV }(\%)\end{array}$ & $111 / 113,99$ \\
\hline Occlusive pressure in PVs with common ostia , $\mathrm{n}$ & $16 / 18$ \\
\hline $\begin{array}{l}\text { Fluroscopy utilization change (25 procedures vs initial ten procedures), } \\
\text { min }\end{array}$ & $21.6 \pm 9.4$ vs $34.4 \pm 10.1$ \\
\hline Contrast utilization change (25 procedures vs initial ten procedures), cc & $23.6 .6 \pm 23$ vs $74 \pm 28$ \\
\hline 12-month arrhythmia free survival after ablation, \% & 60 \\
\hline
\end{tabular}

Figure 5. Representative results.

\section{Abbreviations}

$\mathrm{PV}=$ pulmonary vein, $\mathrm{CBA}=$ cryoballoon ablation, $\mathrm{AF}=$ atrial fibrillation, ICE=intracardiac echocardiography, TEE=transesophageal echocardiography, LA=left atrium

\section{Discussion}

We present a novel technique of direct pulmonary venous pressure monitoring for assessment of complete pulmonary vein occlusion during cryoballoon ablation for atrial fibrillation. We have demonstrated a steep learning curve during application of the direct pressure monitoring technique and a rapid decrease in radioiodine contrast and fluoroscopy utilization after limited training. The technique can be performed with procedure tools commonly available in most laboratories and the setup requires limited additional preprocedure setup time.

Cryoablation utilizing a cryoballoon has emerged as a novel invasive management strategy for the treatment of paroxysmal atrial fibrillation ${ }^{2}$. Procedural success with cryoballoon ablation is highly dependent on correct apposition of the cryoballoon at the pulmonary vein ostium and achievement of complete occlusion of the pulmonary vein ${ }^{6}$. Several predictors of PV occlusion and electrical isolation have been studied: 1 . the minimum temperature achieved during cryoapplication as well as the slope of rewarming are correlated with electrical isolation ${ }^{7}$; however, no precise cut-off values have been assessed and this approach can only be utilized in a retrospective manner and does not allow for confirmation of PV occlusion prior to the initiation of the freezing process 2 . Confirmation of complete occlusion is most commonly assessed by intravenous injection of radioiodine contrast after balloon inflation at the pulmonary vein ostium ${ }^{6}$. Contrast leak upon balloon inflation is assessed fluoroscopically and confirms incomplete pulmonary vein occlusion with the cryoballoon. Multiple injections are frequently required upon cryoballoon repositioning resulting in repeated patient exposure to radioiodine contrast and radiation. As such, contrast may be contraindicated in patients with chronic kidney disease or known allergic reaction to iodine. Lack of continuous feedback during initial balloon positioning and inflation is an added disadvantage of fluoroscopic assessment for PV occlusion 3. Lastly, TEE-guided balloon positioning has been well 
described and allows for direct visualization of the antral position of the cryoballoon and continuous reassessment of balloon positioning and PV occlusion with saline infusion ${ }^{8}$ as well as evaluation for "leaks" with color flow Doppler; however this technique requires continuous utilization of transesophageal echocardiography under general anesthesia, potentially increasing the risk for periprocedural complications associated with general anesthesia and espophageal intubation.

We observed a steep learning curve allowing for rapid and consistent interpretation of pressure waveforms after limited initial experience. Contrast utilization is directly negatively correlated with acquiring expertise in this technique and utilization of fluoroscopy is also dramatically reduced after initial training on pressure monitoring.

The pressure monitoring described surpasses the pitfalls mentioned above and allows for accurate and continuous assessment of PV occlusion. Currently, one balloon design is available for clinical use (Medtronic Inc., MN). This system includes an over-the-wire balloon catheter, which is cooled using nitrous oxide. Contrast is typically injected via an inner lumen; for the purpose of our described method of pressure monitoring, this inner lumen is utilized for direct assessment of PV pressure with the utilization of a single pressure transducer. As such, other than a single 3way manifold, no extra catheters are necessary for application of this method. Further, if necessary, contrast may still be utilized if needed for further confirmation of PV occlusion, though we have demonstrated in a single center study that contrast utilization is largely unnecessary once the pressure monitoring technique has been established.

Pressure waveforms are obtained via the pressure transducer connected to the inner lumen and are displayed on the screen. The balloon is further advanced to the pulmonary vein ostium and inflated as done during standard cryoablation; no adjustments in the standard balloon manipulation approach are necessary when the technique of pressure monitoring is applied. Continuous monitoring for acute dislodgement during the initial freezing period is also possible with the pressure monitoring technique, until a measured scavenged gas temperature drop below -10 degrees Celsius. Particularly, balloon dislodgement from the PV ostium is usually observed upon initial refrigerant injection with resultant balloon expansion ("pop-out" phenomenon) and refinement of balloon positioning can be performed safely if the pressure waveform loses the occlusive pressure characteristics. A similar technique has been utilized in patients presenting in sinus rhythm for cryoballoon ablation

9 . We have observed accurate determination of occlusive pressure in patients with ongoing atrial fibrillation during the procedure.

Currently, two sizes of the cryoballoon catheter are available, $28 \mathrm{~mm}$ and $23 \mathrm{~mm}$ diameter ${ }^{10}$. Usage of the $23 \mathrm{~mm}$ balloon is limited, however the pressure monitoring technique is not affected by balloon size. The pressure monitoring technique is independent of rhythm and is applicable to patients presenting in atrial fibrillation. It is imperative that recording noise is eliminated and accurate zeroing is obtained to allow for accurate detection of the point of occlusive pressure. Displaying systemic arterial pressure as well as coronary sinus (CS) or surface ECG tracings are important to determine "live" the presence of $A$ waves and change in $V$ wave morphology. A common ostium is frequently observed, particularly in the left PVs. We observed occlusive pressure waveforms and complete electrical isolation in 16/18 PV with common ostium. Subselective wiring of the inferior and superior branches can be achieved in the presence of common ostium. Given these results, presence of a common PV ostium is not a limiting factor in the utilization of this technique.

Recurrent AF or atrial flutter was noted in fourteen patients (40\%) after the initial 3 month blanking period and repeat PVI was performed in twelve patients (35\%). Our results are comparable to published literature considering the inclusion of patients with persistent atrial fibrillation as well as six patients who had previously undergone PVI, implying a more extensive AF substrate. Interestingly, recurrences were more common in the first ten cases $(6 / 10)$ despite higher utilization of contrast on initial testing. Of the last fifteen patients studied with minimal contrast utilization (mean contrast $10.9 \mathrm{cc}$ ), four had recurrent AF post procedure indicating that the dramatic reduction in contrast utilization did not adversely influence clinical outcomes.

\section{Limitations}

Given the inability to record a pressure waveform once the intraluminal temperature decreases to less than -10 degrees, late balloon dislocations cannot be detected. This however is unlikely since at this point cryoadherence has occurred. In addition, perturbations are introduced in the pressure waveform during phrenic nerve pacing and pressure recording characteristics become less accurate immediately upon inflation in the right PVs. Atrial fibrillation is not a limiting factor for pressure monitoring application, however it may make the waveform interpretation more difficult for the inexperienced operator. Potential risks associated with air trapped in the 3-way system are limited but not insignificant. However, similar risk for air embolism is posed by utilization of the inner catheter lumen for contrast injection.

In this pilot study, the positive predictive value was noted to be $99 \%$ indicating a high likelihood of complete electrical isolation upon observation of occlusive pressure prior to cryoapplication. In contrast, the negative predictive value was low (40\%) and was driven by the frequency of non occlusive pressure waveforms in the RIPV. In all patients, more than one cryoapplications were performed and as such we cannot exclude the possibility of minimal leaks during each cryoapplication with eventual complete electrical isolation due to refined maneuvering in the vein and apposition of the balloon in previously no ablated regions. This is particularly true for the RIPV in which, due to its relatively steep entrance into the antrum, slight changes in balloon orientation are required to achieve effective cryoablation in a segmental approach. As such, pressure monitoring may not be as helpful in the RIPV given this anatomical variation.

In our series, we observed lack of electrical vein isolation despite demonstration of occlusive pressure in two veins. There are several plausible explanations for this discrepancy, including the presence of epicardial muscle fibers between the antrum and the pulmonary vein or balloonvein mismatch that results in ineffective cryoablation in part of the tissue. This phenomenon occurred during utilization of the first generation cryoballoon and is highly unlikely to occur with the imminent availability of the cryoballoon Advance, which will allow more equal distribution of cryoenergy at the PV ostium. Finally, the technique was primarily applied to patients undergoing cryoballoon ablation under conscious sedation, however assessment of the pressure waveform at end-expiration in the intubated patient should theoretically provide the same accurate results. We have observed consistent results in a small subset of patients undergoing general anesthesia, though this subgroup was not included in the analysis presented here. 


\section{Conclusions}

The technique of pressure assisted PV isolation is simple, reproducible and safe. It significantly reduces the contrast burden and radiation exposure. Since the pressure can be recorded after the freezing process starts, the risk of loss of occlusion in the initial several seconds of balloon cooling (due to "pop-out" phenomenon) is minimized. Fine adjustments of balloon positioning can be safely made within the first 10 sec of cooling before the freezing occur.

\section{Disclosures}

Production and Free Access to this article is sponsored by Medtronic Inc.

Drs. Dan and Wickliffe are consultants for Medtronic Inc. Brian Jones is an employee of Medtronic Inc. Other authors have no conflict of interest to declare.

\section{Acknowledgements}

Funding was obtained by Medtronic Inc.

\section{References}

1. Pappone, C., Rosanio, S., Oreto, G., et al. Circumferential radiofrequency ablation of pulmonary vein ostia: A new anatomic approach for curing atrial fibrillation. Circulation. 21, 102 (21), 2619-28 (2000).

2. Piccini, J.P. \& Daubert, J.P. Cryoablation of atrial fibrillation. J. Interv. Card. Electrophysiol. 32 (3), 233-42 (2011).

3. Kühne, M., et al. Cryoballoon versus radiofrequency catheter ablation of paroxysmal atrial fibrillation: biomarkers of myocardial injury, recurrence rates, and pulmonary vein reconnection patterns. Heart Rhythm. 7 (12), 1770-6 (2010).

4. Kojodjojo, P., O'Neill, M.D., Lim, P.B., et al. Pulmonary venous isolation by antral ablation with a large cryoballoon for treatment of paroxysmal and persistent atrial fibrillation: medium-term outcomes and non-randomized comparison with pulmonary venous isolation by radiofrequency ablation. Heart. 96 (17), 1379-84 (2010).

5. Chao, T.F., Lin, Y.J., \& Chang, S.L. Associations between renal function, atrial substrate properties and outcome of catheter ablation in patients with paroxysmal atrial fibrillation. Circ J., 75 (10), 2326-32 (2011).

6. Van Belle, Y., Janse, P., Rivero-Ayerza, M.J., et al. Pulmonary Vein Isolation Using an Occluding Cryoballoon for Circumferential Ablation: Feasibility, Complications, and Short-term Outcome. Eur. Heart J. 28 (18), 2231-7 (2007).

7. Fürnkranz, A., Köster, I., Chun, K.R., et al. Cryoballoon temperature predicts acute pulmonary vein isolation. Heart Rhythm. 8 (6), $821-5$ (2011).

8. Siklody, C.H., Minners, J., Allgeier, M., et al. Cryoballoon pulmonary vein isolation guided by transesophageal echocardiography: Novel aspects on an emerging ablation technique. J. Cardiovasc. Electrophysiol. 20 (11), 1197-202 (2009).

9. Siklódy, C., Minners, J., Allgeier, M., et al. Pressure-guided Cryoballoon Isolation of the Pulmonary Veins for the Treatment of Paroxysmal Atrial Fibrillation. J. Cardiovasc. Electrophysiol. 21 (2), 120-5 (2010).

10. Chierchia, G.B., de Asmundis, C., Sorgente, A., et al. Anatomical extent of pulmonary vein isolation after cryoballoon ablation for atrial fibrillation: comparison between the 23 and 28 mm balloons. J. Cardiovasc. Med. 12 (3), 162-6 (2011). 